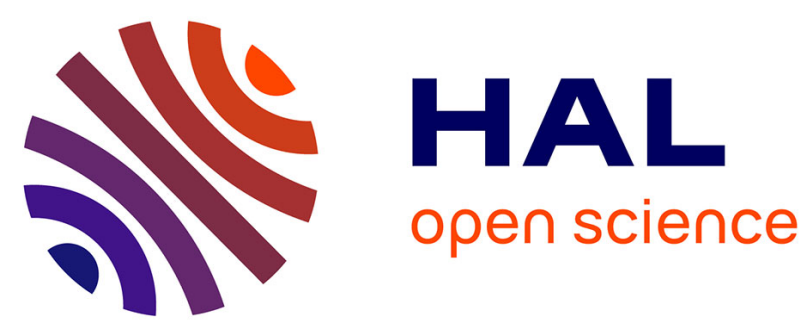

\title{
Multi-domain Modeling and Simulation of an Oximeter: PVT Variations Impact of Opto-electronic Devices on the SpO2 Quantification
}

\author{
Songlin Li, Julien Denoulet, Olivier Tsiakaka, Sylvain Feruglio
}

\section{To cite this version:}

Songlin Li, Julien Denoulet, Olivier Tsiakaka, Sylvain Feruglio. Multi-domain Modeling and Simulation of an Oximeter: PVT Variations Impact of Opto-electronic Devices on the SpO2 Quantification. 11th International Conference on Simulation and Modeling Methodologies, Technologies and Applications, Jul 2021, Online Streaming, France. pp.303-310, 10.5220/0010548903030310 hal-03301569

\section{HAL Id: hal-03301569 \\ https://hal.science/hal-03301569}

Submitted on 27 Jul 2021

HAL is a multi-disciplinary open access archive for the deposit and dissemination of scientific research documents, whether they are published or not. The documents may come from teaching and research institutions in France or abroad, or from public or private research centers.
L'archive ouverte pluridisciplinaire HAL, est destinée au dépôt et à la diffusion de documents scientifiques de niveau recherche, publiés ou non, émanant des établissements d'enseignement et de recherche français ou étrangers, des laboratoires publics ou privés. 


\title{
Multi-domain modeling and simulation of an oximeter: PVT variations impact of opto-electronic devices on the $\mathrm{SpO}_{2}$ quantification
}

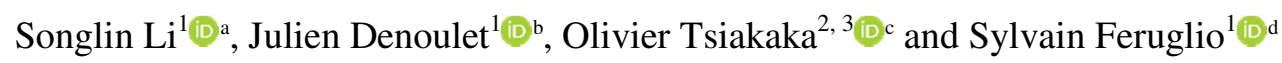 \\ ${ }^{1}$ CNRS UMR 7606, LIP6, Sorbonne Université, Paris, France \\ \{songlin.li, julien.denoulet, sylvain.feruglio\}@lip6.fr \\ ${ }^{2}$ CERVO, University of Laval, Quebec, Canada \\ ${ }^{3}$ Biomedical Microsystems Laboratory, Université Laval, Quebec, Canada \\ olivier.tsiakaka.1@ulaval.ca
}

Keywords: Heterogeneous system, modeling, Monte-Carlo simulation, multi-domain, Near-Infrared Spectroscopy (NIRS), oxygen saturation $\left(\mathrm{SpO}_{2}\right)$, PhotoPlethysmoGraphy (PPG), PVT (Process, Voltage, Temperature), SystemC(-AMS).

Abstract: Oxygen concentration quantification in the blood $\left(\mathrm{SpO}_{2}\right)$ has been used as a mean to diagnose and prevent critical medical conditions thanks to pulse oximetry. In spite of its theoretical precision, this method suffers from intrinsic deviations from the components used in such monitoring devices (PVT) that can lead to $\mathrm{SpO}_{2}$ measurement errors. In this paper, we propose a multi-domain modeling of a NIRS-based blood oxygen saturation monitoring system and its biological environment using SystemC(-AMS) for virtual prototyping, to analyze the impact of PVT variations of opto-electrical components, thanks to Monte-Carlo simulation correlated with transient analysis. We simulated the blood flow of the finger tissue and the dynamic attenuation of the red and infrared light passing through the tissue. The Monte-Carlo simulation method was used to analyze different PVT parameters that may cause measurement deviations separately. Finally, we found that the red/IR LED peak wavelength deviation and the temperature of the system have an important impact on the $\mathrm{SpO}_{2}$ quantification, especially red LED peak wavelength deviation. This result shows that the choice of the red light source is of prime importance for accurate $\mathrm{SpO}_{2}$ quantification.

\footnotetext{
https://orcid.org/0000-0003-4717-1328

https://orcid.org/0000-0003-1354-961X

https://orcid.org/0000-0001-8723-7825

diD https://orcid.org/0000-0002-6444-2671
} 


\section{INTRODUCTION}

$\mathrm{SpO}_{2}$ (oxygen saturation) index is measured to show the level of oxygenation in blood using an oximeter device. It is based on Near-InfraRed Spectroscopy (NIRS) and on PhotoPlethysmoGraphy (PPG) technologies (Webster, 1997). Typically, as shown in Fig.1, two colored light sources (red and infrared) are used on body parts, usually a finger, to detect volumetric changes of blood. However, deviations always occur when performing a measurement. Therefore, device calibration is indispensable to obtain an acceptable error rate (Maxim, 2019).

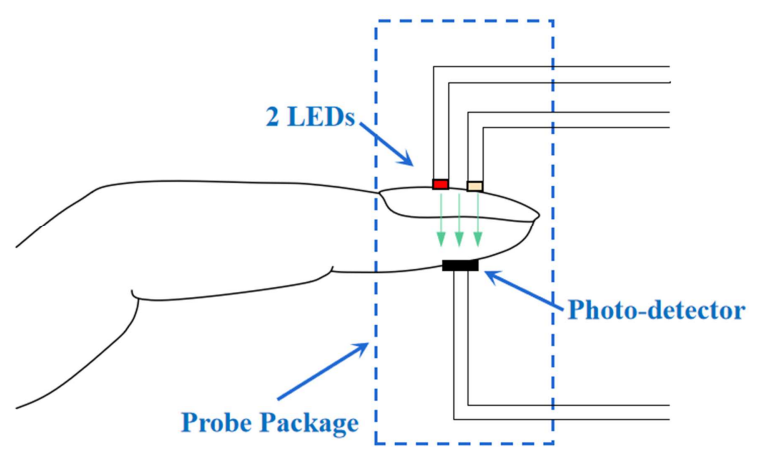

Figure 1: The principle of oximeter.

Many well-known factors have an impact on the $\mathrm{SpO}_{2}$ measurement, for instance motion artifacts, ambient light, skin color, etc. However, so far, influences coming from the system itself haven't been carefully studied. We propose to contribute to this field through the study of the impact of PVT (Process, Voltage, Temperature) variations in the $\mathrm{SpO}_{2}$ monitoring system. To do this, we intend to model and simulate the embedded system in association with the monitored biological tissue.

Evaluating PVT variations is difficult to achieve through experimental methods, because it is not easy to vary certain parameters in the monitoring system (such as the peak wavelength of the red/IR LED spectrum, the spectral sensitivity of the photo- detector, etc.) to evaluate their impact on $\mathrm{SpO}_{2}$ quantification. Parasitic signals and noise might also influence the results. Therefore, we will add these features in our modeling methodology, to give us a better knowledge of the measurement device.

We chose the SystemC and SystemC-AMS modeling tools to develop our models due to three main advantages compared to other modeling tools/languages, which are summarized in Table 1:

- Multi models of computation: SystemC/ SystemC-AMS offer several Models of Computation (MoC) with Discrete Event (DE), Timed Data Flow (TDF), Linear Signal Flow (LSF) and Electrical Linear Networks (ELN). Each MoC operates at different abstraction levels and can therefore be chosen according to the desired precision;

- Fast simulation: with well-chosen abstraction level and simulation time step, simulation run time can be shortened as much as possible;

- Open source: SystemC and SystemC-AMS are freely distributed $\mathrm{C}++$ libraries, which means we have access to these tools as well as all $\mathrm{C}++$ supported functions. If necessary, it is also relatively easy to integrate other software in our model by implementing interfaces.

Other advantages, like high data accuracy, lightweight installation and simplicity of usage, are also reasons why we chose this tool. We used versions 2.3.2 of SystemC and 2.1 of SystemCAMS. Both of these libraries can be freely downloaded on Accellera's website (Accellera, n.d.).

SystemC/SystemC-AMS was proposed as an efficient tool for modeling complex systems, especially in the biomedical domain (Pecheux, et al., 2010), but it doesn't natively include Monte-Carlo (MC) statistical analysis method (Menčík, 2016). Indeed, this analysis is particularly relevant to observe the impact of key parameters on the system behavior. Therefore, a MC simulation method in SystemC-AMS must be developed.

Nowadays, modeling and simulation analysis methods have been widely used in the field of bio-

Table 1: SystemC/SystemC-AMS versus other modeling tools.

\begin{tabular}{|c|c|c|c|c|}
\hline Parameters & VHDL-AMS/Verilog-A & SystemC/SystemC-AMS & SPICE & Comsol/Ansys \\
\hline Accuracy & \multicolumn{2}{|c|}{ Medium - High } & \multicolumn{2}{|c|}{ High } \\
\hline Abstraction level & \multicolumn{2}{|c|}{ Multi-abstraction level } & $\begin{array}{c}\text { Transistor /Netlist } \\
\text { level }\end{array}$ & $\begin{array}{c}\text { Low abstraction } \\
\text { level }\end{array}$ \\
\hline Time to model & Less to Medium & Less & \multicolumn{2}{|c|}{ High } \\
\hline Simulation run time & \multicolumn{2}{|c|}{$\begin{array}{c}\text { Fast - Medium } \\
\end{array}$} & \multicolumn{2}{|c|}{ Medium - Low } \\
\hline Tools required & Compiler/Simulator & $\begin{array}{c}\text { AMS libs with } \\
\text { GCC/Simulators }\end{array}$ & Compiler/Simulator & Simulator \\
\hline Support & Available & Few & \multicolumn{2}{|c|}{ Available } \\
\hline Usage complexity & \multicolumn{3}{|c|}{ Less to Medium } & High \\
\hline Open source & \multicolumn{3}{|c|}{ Possible } & No \\
\hline
\end{tabular}




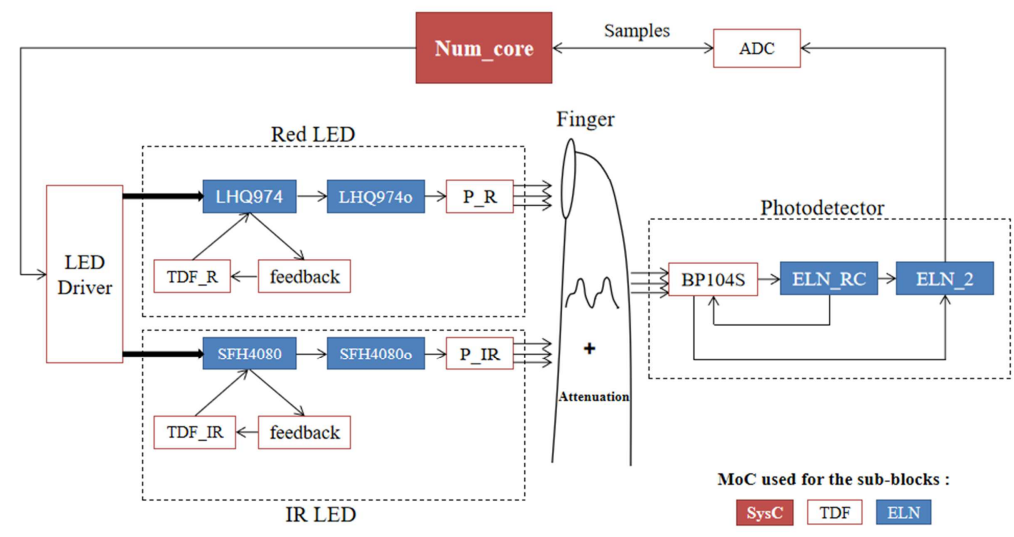

Figure 2: Model structure to emulate oximeter on finger in SystemC/SystemC-AMS.

medicine (e.g. bionic nervous systems modeling (Cacciapuoti, 2015)) and the research of medical device (Mundt, 2000). The interaction modeling between medical device and the human body also exists, such as the interaction between ears and cochlear implants (Tran, 2015), and the interaction simulation between the cardiac rhythm and pacemakers (Greenhut, 1993).

In this context, the main objective of this article is to present our modeling and simulation approach of a highly multi-domain application with opensource tools, and to explore impacts of PVT variations in this biomedical device.

The paper is organized as follows. In section 2 , we present our model structure. Then, the simulation process and the model validation method are shown in section 3. PVT variation analysis is described in section 4. Finally, we conclude in the last section.

\section{MODEL STRUCTURE}

There are mainly two parts in our model: the monitoring system, with opto-electrical components and the biological environment. Figure 2 shows the model block diagram (with the MoC used for each sub_block), with the finger as the monitored biological medium.

In this figure, Num_core block was modeled in SystemC, and a state machine was implemented to emulate a micro-controller. This block was also used to provide pulsed power supply for both LEDs by controlling a $L E D$ Driver, and to receive data from the $A D C$ channel.

\subsection{LED}

A red LED (LHQ974) and an IR LED (SFH4080) manufactured by OSRAM were modeled in
SystemC-AMS (note that any other type of light source can subsequently be modeled, as long as parameters describing it are available). Both LED models are similar, except for their optical and electronic parameters. The LED model is divided into five sub-blocks to simulate static and dynamic behaviors, in association with its optical characteristics. Sub-block $T D F_{-} R$, simulates the static behavior described by Shockley equation (Sze, et al., 2006), mainly. Blue sub-blocks are implemented with the ELN MoC to simulate the dynamic behavior of the LED. Figure 3 (left) shows the physical equivalent circuit of both $L H Q 974$ and $S F H 4080$ sub-blocks. In this equivalent circuit, $C_{d}$ and $C_{j}$ represent the diffusion and the junction capacitance, respectively. $i_{D O}$ is the equivalent current source, which takes the value of the output current of $T D F_{-} R$ and $T D F_{-} I R$. The feedback subblocks are used to transfer two feedback parameters: the current $i_{D}$ and the voltage $V_{D}$ of the LED, from $L H Q 974$ to $T D F \_R$ or from $S F H 4080$ to $T D F \_I R$. Figure 3 (right) represents the equivalent circuit of LHQ974o and SFH4080o, which are used to take into account the intrinsic opto-electrical effect of the device. $i_{\text {equ }}$ is equal to $i_{D}$. The RC pole, represented by the resistance $R_{R C}$ and the capacitance $C_{R C}$, designates the frequency characteristic of the LED (Bian, et al., 2008). Then, the $P_{-} R$ and $P_{-} I R$ subblocks are used to convert the output current $i_{R}$ of LHQ974o and SFH4080o into luminous intensity in Watt. The actual spectral distribution of both LEDs is usually modeled by a Gaussian curve (Tsiakaka, et al., 2020) and the total radiant flux is the integral of the entire spectrum.

For the oxygen rate calculation, since the molar extinction $\varepsilon(\lambda)$ varies according to the wavelength, the attenuation for different wavelengths of light is calculated separately, when a beam of light passes through the biological tissue. Therefore, we should 
separate the luminous intensity of each wavelength in the LED model. Still, we cannot consider all wavelengths of the LED spectrum, since it would greatly increase the amount of calculation and, therefore, the simulation time. As a result, we approximated the LED spectrum by dividing it into five intervals around the peak wavelength. For the red LED (LHQ974), the peak wavelength is ideally $660 \mathrm{~nm}$. Thus, we chose five wavelengths from $640 \mathrm{~nm}$ to $680 \mathrm{~nm}$ with an interval of $10 \mathrm{~nm}$. For the IR LED (SFH4080), with its peak at $880 \mathrm{~nm}$, the chosen range was $860 \mathrm{~nm}$ to $900 \mathrm{~nm}$, with the same interval.

\subsection{Photo-detector}

A model of the BP104S photo-detector (PD), also manufactured by OSRAM, was developed. The PD operates in reverse bias. It provides a spectral sensitivity in accordance with the spectrum of the received light to convert the light intensity into electric power. The current generated by the PD mainly consists of two parts:

- $\mathrm{I}_{\mathrm{ph}}$ : the photonic current generated by the light received from the external environment by the PD, which carries the detection information;

- $\mathrm{I}_{\mathrm{dc}}$ : the parasitic dark current generated by the PD in the absence of wanted light.

Sub-block BP104S in Fig. 2 is used to describe the PD spectral sensitivity and the dark current. $E L N \_R C$ and $E L N \_2$ are made to simulate the dynamic behavior, as in Fig. 3. The PD output is sent to the $A D C$ block, so that the pulsed current can be sampled, after amplification and voltage conversion, and data can be registered in Num_core.

\subsection{Biological environment}

A model of a finger was constructed to simulate the oxygen concentration variation in the blood and its detection with lights through the tissue. In the blood, there are mainly two chromophores that impact the measurement of $\mathrm{SpO}_{2}$ : $\mathrm{HbO}_{2}$ (oxy-hemoglobin) and $\mathrm{HHb}$ (deoxy-hemoglobin). The light attenuation by the biological tissue is given by the Beer-Lambert Law, shown in equation (1):

$$
I(\lambda)=I_{0}(\lambda) \cdot e^{-\left(\varepsilon_{H b O_{2}} \cdot\left[H b O_{2}\right]+\varepsilon_{H H b}[H H b]\right) \cdot d}
$$

with $\mathrm{I}_{0}(\lambda)$ the input light intensity at the wavelength $\lambda, \mathrm{I}(\lambda)$ the output light intensity passing through and attenuated by the biological tissue, $\varepsilon_{\mathrm{HbO} 2}, \varepsilon_{\mathrm{HHb}}$ the molar extinction of $\mathrm{HbO}_{2}$ and $\mathrm{HHb}$, respectively,

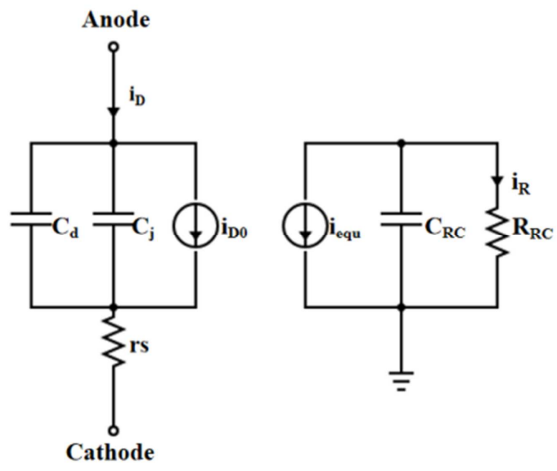

Figure 3: Equivalent circuit of LED in dynamic mode. On the left is the physical equivalent circuit. The block on the right has no physical reality, but is used to consider the device intrinsic opto-electrical effect.

$\left[\mathrm{HbO}_{2}\right]$ and $[\mathrm{HHb}]$ the concentration of $\mathrm{HbO}_{2}$ and $\mathrm{HHb}$, and d the length of optical path.

The change of blood volume according to time is simulated by the normalized equation $(2)$, where $f_{0}$ is the cardiac frequency in $\mathrm{Hz}$.

$$
A=\frac{1}{2} \cdot \sin \left(2 \pi \cdot f_{0} \cdot t\right)+\frac{1}{4} \cdot \sin \left(2 \pi \cdot 2 f_{0} \cdot t\right)
$$

\section{SIMULATION PROCESS AND MODELS VALIDATION}

This section first introduces the simulation process. Then, in subsection 3.2, the accuracy of our models (at the device and at the system level) is evaluated by comparing our simulation with other experimental results in the existing literature.

\subsection{Simulation process}

Once all models were implemented, basic transient simulation was performed to get two PPG signals that represent the red and IR responses generated by the PD after passing through the biological medium. Then, as shown in Figure 2, data was sampled every $1 \mathrm{~ms}$ by the $A D C$ block. The result is presented in Figure 4, where inputs for each LED are light pulses of $150 \mu \mathrm{s}$ every $1 \mathrm{~ms}$. The simulation step was set to $1 \mu \mathrm{s}$, to be able to observe the devices transient behaviors. The heart rate was set to $10 \mathrm{~Hz}$ (10 times higher than in reality), to reduce the simulation time. In this figure, the pink signal is the pulsed current generated by the PD. The top envelope corresponds to the IR LED, while the bottom envelope is due to the red LED. The red LED signal amplitude is lower because the luminous intensity of red light is 


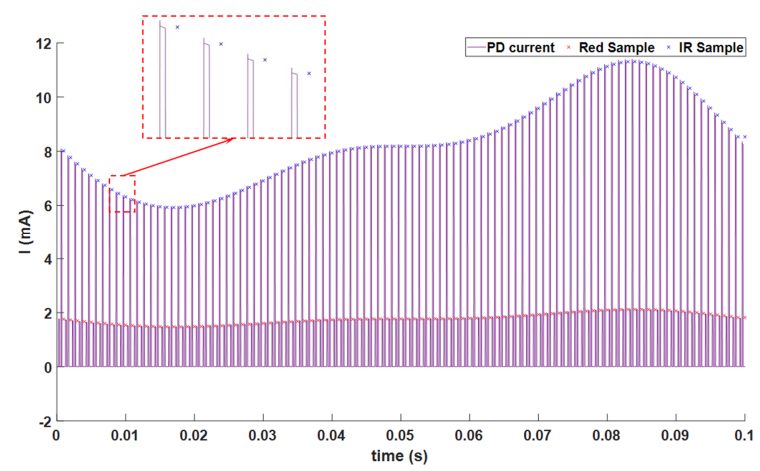

Figure 4: Red signal (bottom), IR signal (top) generated by photo-detector and the samples of the signals (crosses on the top and on the bottom).

relatively weaker, when the same voltage is applied to both LEDs. The crosses (in the zoomed block) are samples of LED signals. On a laptop with an Intel Core i5 $9^{\text {th }}$ Gen processor $(2.4 \mathrm{GHz}, 8 \mathrm{CPUs})$ and $8192 \mathrm{M}$ of RAM, it takes $29 \mathrm{~s}$ to simulate a $0.1 \mathrm{~s}$ duration.

Then, after detecting the peak and valley of the red/IR signals by signal processing (filtering and extremum detection, mainly), we can calculate the $\mathrm{R}_{\mathrm{OS}}$ (Ratio-of-ratio) value with the following equation (Webster, 1997):

$$
R_{O S}=\frac{\ln \left(\frac{R_{L}}{R_{H}}\right)}{\ln \left(\frac{I R_{L}}{I R_{H}}\right)}
$$

Here, $R_{L}, R_{H}$ are the valley and peak value of the red signal, respectively, and $\mathrm{IR}_{\mathrm{L}}, \mathrm{IR}_{\mathrm{H}}$ correspond to the valley and peak value of the IR signal.

$\mathrm{SpO}_{2}$ value was set before simulation run-time, by setting $\left[\mathrm{HbO}_{2}\right]$ and $[\mathrm{HHb}]$ in the biological environment, according to equation (4) (Webster, 1997). We set $\left[\mathrm{HbO}_{2}\right]+[\mathrm{HHb}]=0.3 \mathrm{mM}$, which is an approximate value for the human body (Dash, et al., 2010). In any case, in the simulation, the value of $\mathrm{SpO}_{2}$ can be easily and dynamically changed by modifying the ratio of $\left[\mathrm{HbO}_{2}\right]$ and $[\mathrm{HHb}]$.

$$
\mathrm{SpO}_{2}=\frac{\left[\mathrm{HbO}_{2}\right]}{\left[\mathrm{HbO}_{2}\right]+[\mathrm{HHb}]} \times 100 \%
$$

With a single period of both PPG (red and IR responses), only one $\mathrm{R}_{\mathrm{OS}}$ value can be obtained for one specific $\mathrm{SpO}_{2}$ value. However, to obtain a quadratic $\mathrm{SpO}_{2}-\mathrm{R}_{\mathrm{OS}}$ curve (Maxim, 2019), we need to vary this $\mathrm{SpO}_{2}$ value. In our simulation, we varied this parameter from $90 \%$ to $100 \%$ (a below $90 \%$
$\mathrm{SpO}_{2}$ value means the person is in a danger state). Figure 5 shows several simulation periods. Red lines correspond to PPG due to the red source for three different $\mathrm{SpO}_{2}$ settings $(90 \%, 95 \%$, and $100 \%)$. For IR responses (blue curves), a shift of the peak wavelength has also been taken into account, to illustrate PVT variation, albeit in a basic manner. We chose four values above and four values below the $880 \mathrm{~nm}$ nominal value, each with a $5 \mathrm{~nm}$ step increment, to constitute nine peak wavelength values in the $[860 \mathrm{~nm} ; 900 \mathrm{~nm}]$ interval. Combined with the three different $\mathrm{SpO}_{2}$ parameter values, we were able to simulate twenty-seven configurations. Section 4 will present a more thorough approach to evaluate the influence of PVT variations.

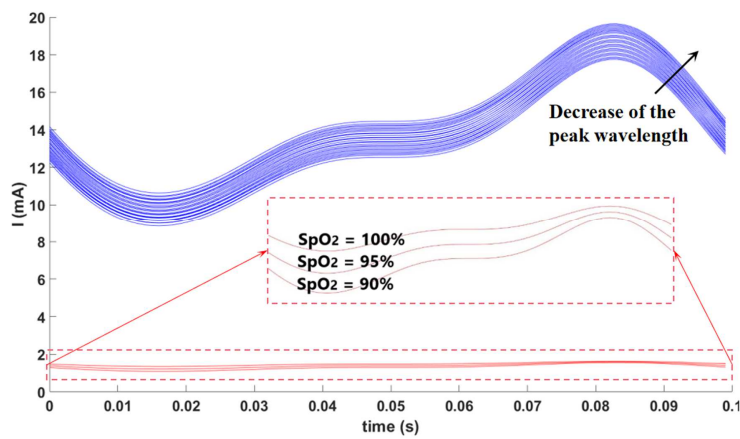

Figure 5: PPG obtained with red and IR sources for three $\mathrm{SpO}_{2}$ value. IR LED curves (in blue) also feature variation of the peak wavelength.

\subsection{Models validation}

There are two steps to validate our models. First, we individually validate each component (i.e. both LEDs and PD) by comparing the simulation output data with key-parameters in the datasheets. These results are summarized in Table 2 (obtained by time domain simulation). Only the optical parameters weren't very well modeled, but with acceptable errors according to datasheets. We assume it was due to approximations made to model certain characterizations and also to the fact that we had the influence of the other parameters. In any case, we can calibrate this error rate by ourselves in the modeling, to be closer to the datasheet value.

The second step is to validate the whole system by comparing our simulation data with previous results (Tsiakaka, et al., 2020), where authors used six different pairs of red/IR LED to plot six $\mathrm{SpO}_{2}$ $\mathrm{R}_{\mathrm{OS}}$ curves. We were able to measure these LEDs optical and electrical characteristics, in order to simulate these devices with our developed model. For the PD, a model of the BP104S was used. As for the biological medium, we applied the same finger 
Table 2: Models key-parameters compared to datasheets.

\begin{tabular}{|c|c|c|c|c|c|}
\hline Components & $\begin{array}{c}\text { Parameters } \\
\text { (Symbol) }\end{array}$ & I/O & $\begin{array}{c}\text { Theoretical } \\
\text { values }\end{array}$ & $\begin{array}{l}\text { Model } \\
\text { values }\end{array}$ & $\begin{array}{l}\text { Drift } \\
\text { rates }\end{array}$ \\
\hline \multirow{4}{*}{$\begin{array}{c}\text { Red LED } \\
\text { (LHQ974) }\end{array}$} & $\begin{array}{c}\text { Forward } \\
\text { voltage }\left(\mathrm{V}_{\mathrm{F}}\right)\end{array}$ & In & $1.8 \mathrm{~V}$ & $1.8 \mathrm{~V}$ & $0 \%$ \\
\hline & \begin{tabular}{|c|} 
Forward \\
current $\left(\mathrm{I}_{\mathrm{F}}\right)$
\end{tabular} & Out & $20 \mathrm{~mA}$ & $20 \mathrm{~mA}$ & $0 \%$ \\
\hline & $\begin{array}{c}\text { Power } \\
\text { consumption } \\
\left(\mathrm{P}_{\mathrm{tot}}\right)\end{array}$ & Out & $40 \mathrm{~mW}$ & $40 \mathrm{~mW}$ & $0 \%$ \\
\hline & $\begin{array}{c}\text { Luminous } \\
\text { intensity }\left(I_{V}\right)\end{array}$ & Out & $15 \mathrm{mcd}$ & $18.0 \mathrm{mcd}$ & $20 \%$ \\
\hline \multirow{4}{*}{$\begin{array}{l}\text { Infra-red LED } \\
\text { (SFH4080) }\end{array}$} & $\begin{array}{c}\text { Forward } \\
\text { voltage }\left(\mathrm{V}_{\mathrm{F}}\right)\end{array}$ & In & $1.5 \mathrm{~V}$ & $1.5 \mathrm{~V}$ & $0 \%$ \\
\hline & $\begin{array}{c}\text { Forward } \\
\text { current }\left(\mathrm{I}_{\mathrm{F}}\right)\end{array}$ & Out & $100 \mathrm{~mA}$ & $104 \mathrm{~mA}$ & $4 \%$ \\
\hline & \begin{tabular}{|c|} 
Power \\
consumption \\
$\left(\mathrm{P}_{\text {tot }}\right)$ \\
\end{tabular} & Out & $250 \mathrm{~mW}$ & $264 \mathrm{~mW}$ & $5.6 \%$ \\
\hline & $\begin{array}{c}\text { Radiant } \\
\text { Intensity }\left(\mathrm{I}_{\mathrm{e}}\right) \\
\end{array}$ & Out & $2.5 \mathrm{~mW} / \mathrm{sr}$ & $2.6 \mathrm{~mW} / \mathrm{sr}$ & $4.4 \%$ \\
\hline PD (BP104S) & $\begin{array}{c}\text { Dark current } \\
\text { (Idark) }\end{array}$ & Out & $2 \mathrm{nA}$ & $2.18 \mathrm{nA}$ & $9 \%$ \\
\hline
\end{tabular}

model. Results are presented in Figure 6.a. It shows the " $110-25 R_{O S}$ " curve, as it is a widely used reference to basically estimate $\mathrm{SpO}_{2}$. We then compared our simulations to Figure 9.b in (Tsiakaka, et al., 2020), with the only difference being the used PD (BP104S for us and FDS100 for the previous work). Figure 6.b shows the $\mathrm{SpO}_{2}$ deviation curves between our models and the previous results, for all six red/IR LED pairs. We can notice that the absolute value of $\Delta \mathrm{SpO}_{2}$ is always less than $1 \%$. Then, we also calculated the $\mathrm{SpO}_{2}$ Root-MeanSquare Deviation (RMSD) for each pair of $\mathrm{SpO}_{2}$ $\mathrm{R}_{\mathrm{OS}}$ curves in both works. It ranges from $0.22 \%$ to $0.77 \%$, which proves that our simulation results correlate well with the previous results. In this way, our whole system model is validated.

\section{PVT VARIATION ANALYSIS}

The dispersion of the IR LED peak wavelength, as simulated in Figure 5, relies on a deterministic approach. For a more in-depth analysis of the impact of PVT variations, it is necessary to introduce a randomness factor. As a result, we performed a MC analysis on our system, based on the variation of selected parameters. As their impact on $\mathrm{SpO}_{2}$ quantification can be rather unpredictable, a Corner simulation will not necessarily give us enough information. Therefore, $\mathrm{MC}$ analysis will allow us to get a more complete view of the system behavior.

\subsection{MC simulation in SystemC-AMS}

In this analysis, we varied selected parameters in a reasonable range, with a chosen Probability Density Function (PDF) of amplitude centered in a specific value. Since this feature isn't natively included in SystemC-AMS, we had to develop our own code. Firstly, we wrote a function to generate a set of numbers varying within a certain range in a specific distribution around 1. In this article, we used a Gaussian distribution. However, other PDF are possible (e.g., uniform). Then, these numbers were multiplied by the nominal value of the parameter to be varied. It constituted a set of input values. Then, we assigned these input values to a SystemC signal and connected it to the systemC-AMS input port of the target parameter. A loop was then implemented in the top file, where we assigned the random input values to the SystemC signal, one by one, to do repeated simulations. Finally, we generated a dat file to save the output data.
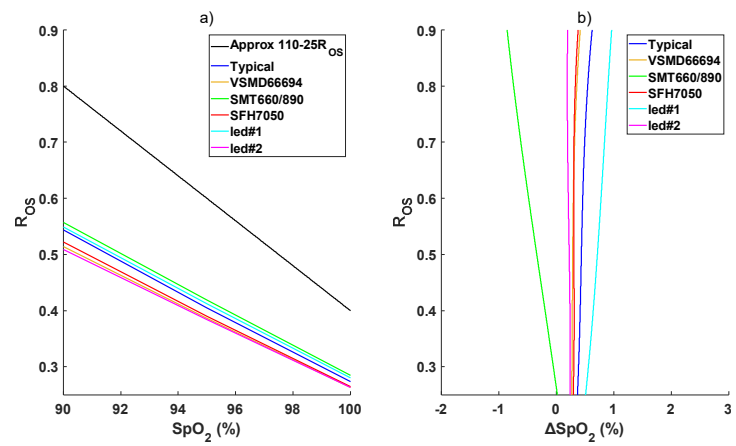

Figure 6: a) Simulation result with our models for the six different red/IR LED pairs; b) $\mathrm{SpO}_{2}$ deviation curves between our works and (Tsiakaka, et al., 2020).

\subsection{Introduction of different PVT parameters in the system}

PVT considers the variation of selected parameters in an electronic system: Process (P) dispersion due to manufacturing, mainly, supply Voltage $(\mathrm{V})$ of the various system components, and the operating Temperature $(\mathrm{T})$ of the device. The parameters we took into account are presented in Table 3, with their typical values and variation ranges.

T-variation in the device is mainly due to selfheating during operation and also to the light sources during emission. Note that a variation of more than $4 \mathrm{~K}$ is generally unacceptable in a medical device. 
Table 3: PVT parameters to be varied and analyzed in the system.

\begin{tabular}{|c|c|c|c|c|c|c|c|c|c|c|c|c|}
\hline \multirow{3}{*}{ Parameters } & \multicolumn{8}{|c|}{ Process (P) } & \multicolumn{3}{|c|}{ Voltage (V) } & \multirow{3}{*}{$\begin{array}{c}\text { Temperature } \\
\text { (T) }\end{array}$} \\
\hline & \multicolumn{2}{|c|}{ Ideality factor (n) } & \multicolumn{2}{|c|}{ Peak wavelength $\left(\lambda_{\text {Peak }}\right)$} & \multicolumn{2}{|c|}{ FWHM } & \multicolumn{2}{|c|}{ Spectral sensitivity of PD $\left(\lambda_{10 \%}\right)$} & \multirow{2}{*}{$\begin{array}{c}\text { Red LED } \\
\left(\mathrm{V}_{\mathrm{F}}\right)\end{array}$} & \multirow{2}{*}{$\begin{array}{c}\text { IR LED } \\
\left(\mathbf{V}_{\mathrm{F}}\right) \\
\end{array}$} & \multirow{2}{*}{$\mathbf{P D}\left(\mathbf{V}_{\mathrm{R}}\right)$} & \\
\hline & Red LED & IR LED & Red LED & IR LED & Red LED & IR LED & lateral & vertical & & & & \\
\hline Typical value & 1,77 & 1,22 & $660 \mathrm{~nm}$ & $880 \mathrm{~nm}$ & $15 \mathrm{~nm}$ & $45 \mathrm{~nm}$ & 1 & 1 & $1.8 \mathrm{~V}$ & $1.5 \mathrm{~V}$ & $10 \mathrm{~V}$ & $300^{\circ} \mathrm{C}$ \\
\hline Variation & {$[1,2.55]$} & {$[1,1.64]$} & $\pm 20 \mathrm{~nm}$ & $\pm 20 \mathrm{~nm}$ & $10 \%$ & $10 \%$ & $10 \%$ & $10 \%$ & {$[1.5 \mathrm{~V} ; 2.5 \mathrm{~V}]$} & {$[1.2 \mathrm{~V} ; 1.8 \mathrm{~V}]$} & {$[5 \mathrm{~V} ; 15 \mathrm{~V}]$} & $10 \%$ \\
\hline RMSD of $\mathrm{SpO}_{2}$ & $0 \%$ & $0 \%$ & $10.49 \%$ & $1.57 \%$ & $0.05 \%$ & $0.02 \%$ & $0.02 \%$ & $0 \%$ & $0 \%$ & $0 \%$ & $0 \%$ & $3.19 \%$ \\
\hline
\end{tabular}

A device power supply voltage is often affected by many factors causing instability and fluctuations (IR-drop due to current draw, crosstalk because of electromagnetic interference, etc.). Moreover, in an embedded system with battery, the voltage supply decreases with the battery usage. Such instability may impact the accuracy of our $\mathrm{SpO}_{2}$ measure. In this study, we focused on the power supply of both LEDs and of the PD, knowing that the $\mathrm{V}_{\mathrm{REF}}$ for the ADC doesn't result in any error at all.

P-variation is the deviation of component attributes during fabrication. For both LEDs, $n$ is the ideality factor, a key parameter describing the diode junction and a solar cell's electrical behavior (Hadj, et al., 2018). n can slightly vary during fabrication. As shown in equation (5), it has an influence on the forward current $\mathrm{I}$, which is directly proportional to the optical power of the light source. $\mathrm{V}_{\mathrm{F}}$, the threshold voltage of the LED is also affected by manufacturing dispersion. Both of the parameters can be concatenated in a same Gaussian PDF.

$$
I=I_{S} \cdot e^{\left(\frac{V_{F}}{n \cdot V_{T}}-1\right)}
$$

In equation (5), $\mathrm{I}_{\mathrm{S}}$ is the saturation current, and $\mathrm{V}_{\mathrm{T}}$ the thermal voltage. We found in the datasheets that the maximal $\mathrm{V}_{\mathrm{F}}$ for the red and IR LED is $2.5 \mathrm{~V}$ and $1.8 \mathrm{~V}$, respectively. From simulations, we estimated the maximal value of $\mathrm{n}$ for both LEDs. It is 2.55 and 1.64, respectively, and can't be less than 1 (Sze, et al., 2006). So, we varied $n$ from 1 to 2.55 for the red LED and from 1 to 1.64 for the IR LED.

Deviation of the LED peak wavelength is another relevant parameter. Even for the same type of LED, the peak wavelength of the optical spectrum is subject to deviation due to the fabrication process. We found a typical range of $\pm 20 \mathrm{~nm}$ for the peak wavelength of both LEDs in their datasheets.

The FWHM (Full Width at Half Maximum) parameter of the optical spectrum can also be impacted by process dispersion. Based on experimental results found in (Filippo, et al., 2017), we chose a range of $\pm 10 \%$ of the typical value.

Finally, the PD spectral sensitivity is also affected by an offset due to process variation. Thus, we studied the impact of this factor by shifting the PD optical response spectrum vertically and laterally.

\subsection{Simulation Results}

To get an idea of the influence of each of the parameters presented above, we first performed the MC simulation (consisting of 200 runs), varying only one single parameter. We were then able to calculate for this parameter the maximal $\mathrm{SpO}_{2}$ RMSD for its two extreme values. These results are presented in the last line of Table 3 . We found that the main factors that impact the $\mathrm{SpO}_{2}$ quantification come from the deviation of both LED peak wavelengths (RMSD is $11.31 \%$ for the red LED and $1.79 \%$ for the IR LED) and from temperature (RMSD is $3.19 \%$ ). The gap between both LED RMSD values can be explained by the fact that around the red light band $(660 \mathrm{~nm})$, the slope of molar extinction curves of $\mathrm{HbO}_{2}$ and $\mathrm{HHb}$ is greater than around the IR light band (880nm). The impact of other parameters is negligible.

After that, we investigated the combined influence of several parameters dispersion on the quantification of $\mathrm{SpO}_{2}$. Figure 7 presents the result of the MC simulation correlated with transient analysis. We varied the three key parameters (both LED peak wavelengths and T). We performed 400 runs (i.e., different configurations) to obtain 400 $\mathrm{SpO}_{2}-\mathrm{R}_{\mathrm{OS}}$ curves, as in Figure 7.a. To get these results, the simulation time was around $11 \mathrm{~h}$. Figure 7.b shows the distribution of $\mathrm{SpO}_{2}$ when $\mathrm{R}_{\mathrm{OS}}$ is equal to 0.4. The RMSD of $\mathrm{SpO}_{2}$ for the two extreme cases

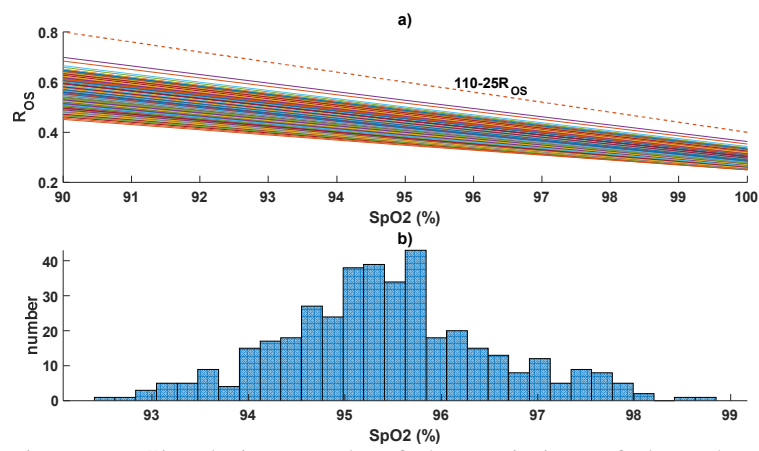

Figure 7: Simulation result of the variation of three key factors in the same time. 
is $9.32 \%$ in the critical $90-100 \%$ saturation window, which is close to the dispersion value associated to the red LED peak wavelength variation. Consequently, it could be said that the red LED peak wavelength variation has the greatest impact.

We can conclude from the above results that in the oximeter manufacturing process, it is necessary to tightly control the peak wavelength deviation of the light source, to avoid an otherwise necessary calibration. At the same time, the device operating temperature influence on the $\mathrm{SpO} 2$ measurement accuracy cannot be ignored. For other parameters of our discussion, there is no strict requirement.

\section{CONCLUSION}

In this paper, the process to quantify $\mathrm{SpO}_{2}$ on the finger with an oximeter is simulated with an optoelectronic model built in SystemC/SystemC-AMS. Then, the impact of PVT variations in the device on the $\mathrm{SpO}_{2}$ quantification is explored, through a $\mathrm{MC}$ method combined with transient simulation, performed on the developed models. We found that the main parameters of PVT variations on the quantification of $\mathrm{SpO}_{2}$ were the red/IR LED peak wavelength deviation and temperature. Other factors seem to have a negligible impact individually.

This modeling method in SystemC/SystemCAMS, which associates the monitoring system, including its opto-electronic components, and the biological environment, is proved fast, accurate and flexible. This methodology can be employed for any cyber-physical system to estimate its performances, to optimize the design phase, and to help the understanding of measurement data (e.g. reproduce results close to the experimental measurements in the simulation and vary certain parameters to understand their impacts).

\section{REFERENCES}

Webster, J.G., ed. 1997. Design of Pulse Oximeters. Bristol; Philadelphia: Institute of Physics Pub, doi: 10.1201/9780367802592.

Maxim, 2019. Guidelines for $\mathrm{SpO} 2$ measurement usig the Maxim MAX32664 sensor hub. https://pdfserv.maximintegrated.com/en/an/SpO2Measurement-Maxim-MAX32664-Sensor-Hub.pdf

Accellera. (n.d.). SystemC. Retrieved February 27, 2021, from https://www.accellera.org/downloads/standards/s ystemc
Pecheux, F., et al., 2010. Is SystemC-AMS an Appropriate "Promoter" for the Modeling and Simulation of BioCompatible Systems?, IEEE ISCAS, doi: 10.1109/ISCAS.2010.5537669.

Menčík, J., 2016. Concise Reliability for Engineers. InTech, doi: 10.5772/62009.

Cacciapuoti, A.S., et al., 2015. Receiver Design for a Bionic Nervous System: Modeling the Dendritic Processing Power. IEEE Internet of Things Journal, 11, doi: 10.1109/JIOT.2015.2438098

Mundt, C.W., et al., 2000. Applications of SPICE for Modeling Miniaturized Biomedical Sensor Systems. IEEE Transactions on Biomedical Engineering, 47(2): 149-54, doi: 10.1109/10.821733.

Tran, P., et al., 2015. Development of HEATHER for Cochlear Implant Stimulation Using a New Modeling Workflow. IEEE Transactions on Biomedical Engineering, 62(2): 728-35, doi: 10.1109/TBME.2014.2364297.

Greenhut, S.E., et al., 1993. A Stochastic Network Model of the Interaction between Cardiac Rhythm and Artificial Pacemaker. IEEE Transactions on Biomedical Engineering, 40(9): 845-58, doi: 10.1109/10.245605.

Bian, J., et al., 2008. A Novel Spice Model of Photodetector for OEIC Design, edited by Liwei Zhou, 66211E. Beijing, China, doi: 10.1117/12.790834.

Tsiakaka, O., et al., 2020. Source-Detector Spectral Pairing-Related Inaccuracies in Pulse Oximetry: Evaluation of the Wavelength Shift, MDPI Sensors, 20(11), doi: 10.3390/s20113302.

Dash, R.K., et al., 2010. Erratum to: Blood $\mathrm{HbO2}$ and $\mathrm{HbCO} 2$ Dissociation Curves at Varied O2, CO2, PH, 2,3-DPG and Temperature Levels. Annals of Biomedical Engineering, 38(4), doi: 10.1007/s10439010-9948-y

Hadj, B., et al., 2018. Theoretical Models for Anomalously High Ideality Factor in a Au/SnO2-Si(n)/Al Solar Cell, Silicon, 10(3), doi: 10.1007/s12633-017-9572-7.

Sze, S.M., 2006. Physics of Semiconductor Devices: Sze/Physics. Hoboken, NJ, USA: John Wiley \& Sons, Inc., doi: 10.1002/9780470068328.

Filippo, R., et al., 2017. LEDs: Sources and Intrinsically Bandwidth-Limited Detectors, MDPI Sensors 17(7), doi: 10.3390/s17071673. 